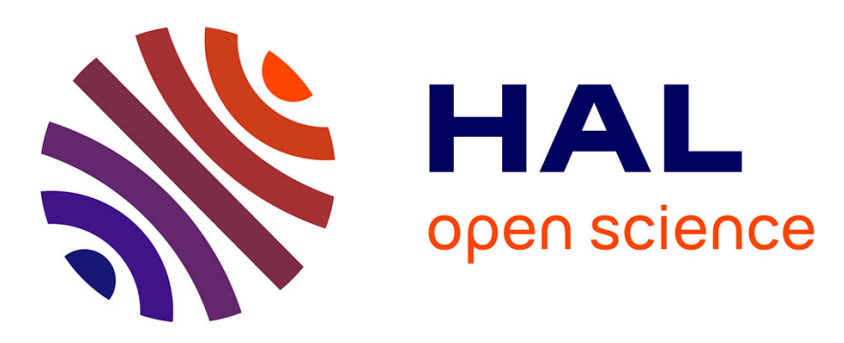

\title{
Physicochemical studies of the interaction of the lipoheptapeptide surfactin with lipid bilayers of L- $\alpha$-dimyristoyl phosphatidylcholine
}

Henny Kell, Josef F. Holzwarth, Christoph Boettcher, Richard K. Heenan, Joachim Vater

\section{To cite this version:}

Henny Kell, Josef F. Holzwarth, Christoph Boettcher, Richard K. Heenan, Joachim Vater. Physicochemical studies of the interaction of the lipoheptapeptide surfactin with lipid bilayers of L- $\alpha$-dimyristoyl phosphatidylcholine. Biophysical Chemistry, 2007, 128 (2-3), pp.114. 10.1016/j.bpc.2007.03.005 . hal-00501657

\section{HAL Id: hal-00501657 https://hal.science/hal-00501657}

Submitted on 12 Jul 2010

HAL is a multi-disciplinary open access archive for the deposit and dissemination of scientific research documents, whether they are published or not. The documents may come from teaching and research institutions in France or abroad, or from public or private research centers.
L'archive ouverte pluridisciplinaire HAL, est destinée au dépôt et à la diffusion de documents scientifiques de niveau recherche, publiés ou non, émanant des établissements d'enseignement et de recherche français ou étrangers, des laboratoires publics ou privés. 


\section{Accepted Manuscript}

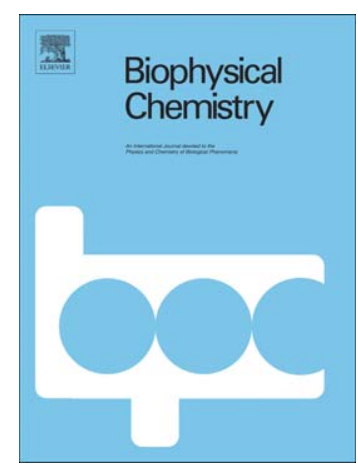

Physicochemical studies of the interaction of the lipoheptapeptide surfactin with lipid bilayers of L- $\alpha$-dimyristoyl phosphatidylcholine

Henny Kell, Josef F. Holzwarth, Christoph Boettcher, Richard K. Heenan, Joachim Vater

PII:

S0301-4622(07)00073-7

DOI:

doi: $10.1016 /$ j.bpc.2007.03.005

Reference:

BIOCHE 4938

To appear in: Biophysical Chemistry

Received date: 20 August 2006

Revised date: $\quad 4$ March 2007

Accepted date: 6 March 2007

Please cite this article as: Henny Kell, Josef F. Holzwarth, Christoph Boettcher, Richard K. Heenan, Joachim Vater, Physicochemical studies of the interaction of the lipoheptapeptide surfactin with lipid bilayers of L- $\alpha$-dimyristoyl phosphatidylcholine, Biophysical Chemistry (2007), doi: 10.1016/j.bpc.2007.03.005

This is a PDF file of an unedited manuscript that has been accepted for publication. As a service to our customers we are providing this early version of the manuscript. The manuscript will undergo copyediting, typesetting, and review of the resulting proof before it is published in its final form. Please note that during the production process errors may be discovered which could affect the content, and all legal disclaimers that apply to the journal pertain. 


\section{Physicochemical studies of the interaction of the lipoheptapeptide surfactin with lipid bilayers of $L$ - $\alpha$-dimyristoyl phosphatidylcholine}

Henny Kell ${ }^{1}$, Josef F. Holzwarth ${ }^{1}$, Christoph Boettcher ${ }^{2}$, Richard K. Heenan ${ }^{3}$ and Joachim Vater $^{4 *}$

${ }^{1}$ Fritz-Haber-Institut, Max-Planck-Society, Faradayweg 4-6, D-14195 Berlin, Germany

${ }^{2}$ Freie Universität Berlin, Forschungszentrum für Elektronenmikroskopie, Fabeckstr. 36a, D14195 Berlin, Germany

${ }^{3}$ ISIS, Rutherford Appelton Laboratories, Chilton, Didcot, OX11 OQX, United Kingdom

${ }^{4}$ Institut für Chemie, Arbeitsgruppe Biochemie und Molekulare Biologie, Technische Universität Berlin, Franklinstr. 29, D-10587 Berlin, Germany

DMPC-surfactin interaction

Correspondence to: Dr. Joachim Vater, Institut für Chemie, Arbeitsgruppe Biochemie und Molekulare Biologie, Technische Universität Berlin, Franklinstr. 29, D-10587 Berlin, Germany; phone: +49-30-31425609; fax: +49-30-31424783; e-mail: vater@,chem.tu-berlin.de 


\section{Abstract}

To understand the biological action of surfactin from Bacillus subtilis we investigated its effects on the phase transition of L- $\alpha$-dimyristoyl phosphatidylcholine (DMPC)-vesicles from the crystalline to the fluid state using differential scanning calorimetry; light scattering; small angle neutron scattering and cryo-electron microscopy. DSC-thermograms revealed two phase transition peaks. Light scattering profiles showed two branches with characteristic hysteresis phenomena. With both techniques the same values of the phase transition temperatures $\mathrm{T}_{\mathrm{m} 1}$ and $\mathrm{T}_{\mathrm{m} 2}$ of 23.5 and $23{ }^{\circ} \mathrm{C}$ were obtained indicating two forms of DMPC-surfactin aggregates which could be visualized by cryo-electron microscopy. Until 4 mol\% surfactin the vesicular form predominated, but was accompanied by bilayered membrane fragments by increasing the biosurfactant concentrations. At surfactin concentrations higher than $15 \mathrm{~mol} \%$ smaller DMPC-surfactin micelles of ellipsoidal conformation were formed, as demonstrated by small angle neutron scattering. In addition, by „Poor Man's“ - temperature jump-relaxation spectroscopy slow transients in the phase transition of vesicular DMPC-surfactin aggregates with relaxation times of 20-30s were detected which presumably indicate the slow dissipation of intermediate lipid- and surfactin domains formed after the main phase transition on the way to the fluid state. This process is accelerated by surfactin.

Keywords: DMPC-vesicles; surfactin; differential scanning calorimetry; static light scattering; cryo-electron microscopy; small angle neutron scattering; „Poor Man’s“ temperature jump-relaxation spectroscopy. 


\section{Introduction}

Microbial biosurfactants, as lipopeptides and glycolipids, show a high potential for medical and biotechnological applications. Surfactin, a lipopeptide which is produced by numerous Bacillus subtilis strains, is one of the most efficient biosurfactants so far known [1,2]. At a concentration of $20 \mu \mathrm{M}(0.05 \%)$ it lowers the surface tension of water from 72 to $27 \mathrm{mN} \mathrm{m}^{-1}$ [1]. It is a cyclic lipoheptapeptide containing seven amino acids and a $\beta$-hydroxy fatty acid of the following structure $[3,4]$ :

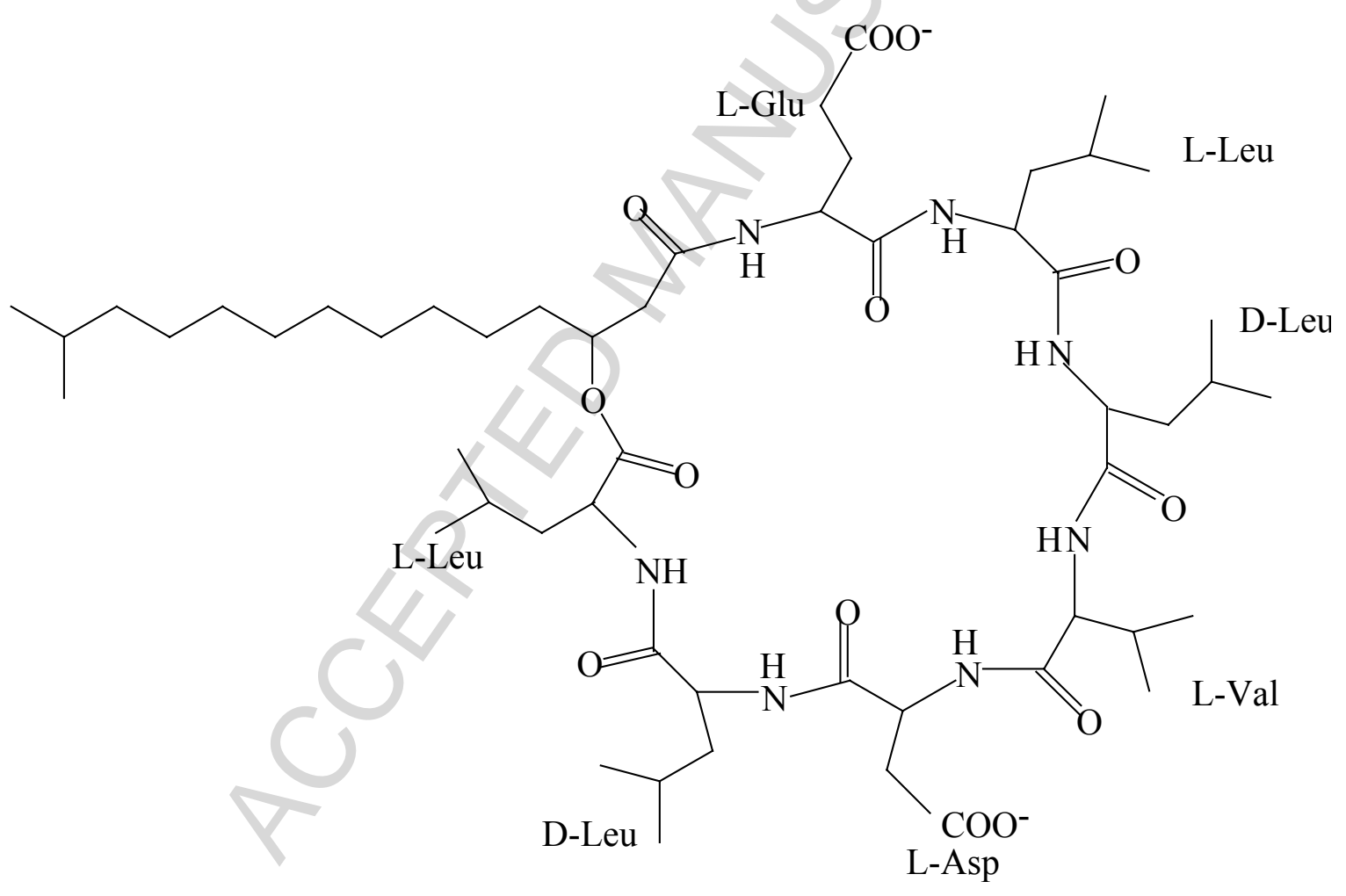

Fig. 1 Structure of surfactin

Ring closure is achieved by lactone formation between the $\beta$-hydroxyl group of the fatty acid component and the carboxyl group of the terminal L-Leu-residue [5]. In solution the peptide ring of surfactin shows a ,horse-saddle“ topology [6]. Its two negatively charged amino acid residues L-Glu and L-Asp form a claw and function as binding sites for mono- and divalent cations, while its fatty acid side chain is extending at the opposite side of the ring. Natural 
surfactin is a complex mixture of closely related isoforms which vary in length and branching of its fatty acid component and/or the amino acid composition and sequence of its peptide ring [7]. It contains $\beta$-hydroxy fatty acids with side chains of 13-15 carbon atoms. Due to its amphiphilic structure it shows unique surface-, interface- and membrane-active properties $[1,2,8-12]$ and a strong tendency for self-aggregation forming micelles and vesicles $[13,14]$. Surfactin is distinguished by a broad spectrum of interesting biological activities which mainly depend on its membrane-active properties. For example, it shows potent antimicrobial [2,10,15], antiviral [16-19], antitumor [20], and hypocholesterolemic [21] activities which are of high relevance in health care and biotechnology. As a membrane-active agent it lyses bacterial spheroplasts and protoplasts [10] as well as mycoplasma and viral membrane envelopes $[15,18]$. It is hemolytic by lysis of erythrocytes inhibiting fibrin clot formation $[10,19]$. Surfactin efficiently binds mono- and divalent cations [22] and forms ion conducting pores in lipid bilayer membranes functioning as an ionophor for ion transport across biomembranes [23,24].

To understand the mechanisms of its specific biological actions detailed knowledge on its structure and physico-chemical properties is needed. It seems obvious that its amphiphilic nature and its specific surface-and membrane-active character play an important role in the expression of its biological activities. Particularly relevant are studies on the interaction of surfactin with artificial model membranes which have been performed by several authors [9,25-29]. In previous work it has been demonstrated by electron microscopic analysis that surfactin inactivates mycoplasma [15] and enveloped viruses [18,19] by purely physicochemical mechanisms. The action of surfactin ultimately leads to complete desintegration of the envelope membranes. Therefore, the physicochemical approach to investigate the effects of surfactin on biomembranes seems most promising. Highly informative to explore the interaction of (bio)membranes with membrane-active compounds, 
as surfactin, are studies of the influence of such agents on the phenomena involved in the temperature dependent phase transition from the crystalline into the fluid state. To clarify the specific action of surfactin we performed such studies with DMPC bilayer model membranes using thermodynamic, kinetic and structural methods. Differential scanning calorimetry (DSC) and light scattering provided evidence for two forms of DMPC-surfactin aggregates which could be visualized as vesicular particles and bilayered lamellar membrane fragments by cryo-electron microscopy, while small angle neutron scattering measurements revealed a third micellar form which presumably represents mixed DMPC-surfactin micelles.

In addition, of high importance is the investigation of the dynamic processes involved in the main phase transition of bilayer membranes and their interaction with membrane-active agents which need the application of fast relaxation techniques. By iodine-laser temperature jump studies in combination with time-resolved cryo-electron microscopic studies Groll et al. [30] discriminated at least five fast processes being involved in the phase transition of lipid bilayer membranes which were observed in a time range of nanoseconds to seconds. In our present study we used the „Poor Man's“ temperature jump technique to investigate slow transients in the time scale of 10-100 seconds which indicate the conversion of intermediate states formed, when the main phase transitions approach the fluid state. 


\section{Materials and Methods}

\section{Materials}

Surfactin was isolated from Bacillus subtilis OKB 105, as published by Baumgart et al. [31]. L- $\alpha$-dimyristoyl phosphatidylcholine (DMPC) and L- $\alpha$-dipalmitoyl phosphatidylcholine (DPPC) were obtained from Sigma-Aldrich (Taufkirchen, Germany).

\section{Preparation of DMPC-vesicles:}

DMPC was dissolved with ethanol in a concentration of $36 \mathrm{mM}$. The solution was slowly injected with a Hamilton syringe into $10 \mathrm{ml} 2.5 \mathrm{mM}$ Tricine buffer, $10 \mathrm{mM} \mathrm{NaCl}$; $\mathrm{pH} 7.5$ at a temperature of $28{ }^{\circ} \mathrm{C}$. The solution of vesicles was dialyzed against the same Tricine buffer for $24 \mathrm{~h}$ at temperatures above the phase transition of the lipids. The vesicle preparation was stable for several days when stored above this temperature.

\section{Methods}

\section{a) Differential scanning calorimetry (DSC)}

DSC-experiments with DMPC vesicles were performed with a MicroCalMC-2 instrument (MicroCal, Northampton, MA, USA) at a lipid concentration of $2.5 \mathrm{mM}$ in $2.5 \mathrm{mM}$ Tricine buffer, $10 \mathrm{mM} \mathrm{NaCl}$; $\mathrm{pH} 7.5$ between 10 and $35{ }^{\circ} \mathrm{C}$. The scan rate was $30{ }^{\circ} \mathrm{C} \mathrm{h}^{-1}$. Heat capacity $\mathrm{c}_{\mathrm{p}}\left(\mathrm{J}^{\circ} \mathrm{C}^{-1}\right)$ was measured as a function of temperature $\left({ }^{\circ} \mathrm{C}\right) . \mathrm{Cp}$ was converted into molar heat capacity $\mathrm{c}_{\mathrm{p}}{ }^{\mathrm{m}}\left(\mathrm{kJ} \mathrm{mol}^{-1}{ }^{\circ} \mathrm{C}^{-1}\right)$ accounting for the cell volume $(\mathrm{V}=1.225 \mathrm{ml})$ and the sample concentration. The two peaks in the DSC curves measured for DMPC were fitted by Gauss functions. The phase transition temperature $\mathrm{T}_{\mathrm{m}}\left({ }^{\circ} \mathrm{C}\right)$ of the DMPC vesicles was obtained from the Gauss curves after baseline subtraction at the highest molar heat capacity $\mathrm{c}_{\mathrm{p}}{ }^{\mathrm{m}}$. The area under the DSC peaks corresponds to the molar enthalpy change $\Delta \mathrm{H}_{\mathrm{cal}}\left(\mathrm{kJ} \mathrm{mol}^{-1}\right)$ for the phase transition. 


\section{b) Static light scattering}

Light scattering measurements on solutions of DMPC-vesicles were performed with a Shimadzu spectrofluorophotometer RF-5000 using a $150 \mathrm{~W}$ Xenon lamp as light source at a wavelength of $300 \mathrm{~nm}$. The scattered light was observed at an angle of $90^{\circ}$. DMPC in a concentration of $2.5 \mathrm{mM}$ was dissolved in $2.5 \mathrm{mM}$ Tricine buffer, $10 \mathrm{mM} \mathrm{NaCl}$; $\mathrm{pH}$ 7.5. Surfactin was injected above the phase transition temperature $T_{m}$ in concentrations between 0.025 and $0.75 \mathrm{mM}(1-30 \mathrm{~mol} \%)$. To study the phase transition phenomena, the intensity of the scattered light was measured as a function of temperature starting with a „Down scan“ in a temperature range of 40 to $15{ }^{\circ} \mathrm{C}$ followed by an „Up scan“ over the same temperature interval. The heating rate was $12{ }^{\circ} \mathrm{C} \mathrm{h}^{-1}$. A Haake F3-C-thermostat was used for the temperature program. Temperature was controlled by thermo-couples of type Therm 2244-1, NTC Typ C, Ahborn Mess- und Regelungstechnik (Holzkirchen, Germany).

\section{c) Cryo-electron microscopy of DMPC-surfactin aggregates}

Electron microscopical investigations were carried out at a DMPC-concentration of $2.5 \mathrm{mM}$ and surfactin concentrations varying between $0.05-0.2 \mathrm{mM}(2-8 \mathrm{~mol} \%)$. Droplets of aqueous sample solutions $(5 \mu \mathrm{l})$ were applied to perforated $(1 \mu \mathrm{m}$ hole diameter) carbon film covered 200 mesh grids (R1/4 batch of Quantifoil Micro Tools GmbH, Jena, Germany), which had been hydrophilized before use by $60 \mathrm{~s}$ plasma treatment at $8 \mathrm{~W}$ in a BALTEC MED 020 device. The supernatant fluid was removed with a filterpaper until an ultra-thin layer $(100-200 \mathrm{~nm})$ of the sample solution was obtained spanning the holes of the carbon film. The samples were immediately vitrified by propelling the grids into liquid ethane at its freezing point $(90 \mathrm{~K})$ operating a guillotine-like plunging device implemented in a humidity and temperature controlled vitrification system [32]. This improved sample preparation technique prevents changes in the sample concentration, which otherwise occurs by 
evaporation of the solvent during preparation, and allows to adjust the sample temperature for measurements above or below the phase transition temperature. The ultrathin vitrified sample layers were subsequently transferred under liquid nitrogen into a Tecnai F20 transmission electron microscope (FEI Company, Oregon, USA) using the Gatan (Gatan Inc., California, USA) cryoholder and -stage (Model 626). Microscopy was carried out at $94 \mathrm{~K}$ sample temperature using the microscopes low dose protocol at a primary magnification of $61702 \times$ and an accelerating voltage of $160 \mathrm{kV}$ (FEG- illumination). The defocus was chosen in all cases to be $1.96 \mu \mathrm{m}$ corresponding to a first zero of the phase contrast transfer function at $2.35 \mathrm{~nm}$.

\section{d) Small angle neutron scattering (SANS) of DMPC-surfactin micelles}

Small angle neutron scattering experiments on a solution of $2.5 \mathrm{mM}$ DMPC in the presence of $0.75 \mathrm{mM}(30 \mathrm{~mol} \%)$ surfactin in Tricine/ $\mathrm{D}_{2} \mathrm{O}$ buffer $\mathrm{pH} 7.5$ were performed with a LOQ diffractometer (ISIS) using a pulsed neutron source in the Rutherford Appleton Laboratories. UK. Measurements were performed at $29{ }^{\circ} \mathrm{C}$ using the „,white beam-time of flight“ - method. Incident neutron wavelengths in the range of $2-10 \AA$ were used at a frequency of $50 \mathrm{~Hz}[33]$. The distance between sample and detector was $5 \mathrm{~m}$. The scattering directions $\mathrm{Q}$ varied between 0.02 and $0.25 \AA^{-1}$. Prior to the measurements a temperature treatment of the DMPCsurfactin solution in the range of $15-40{ }^{\circ} \mathrm{C}$ in up- and down-mode was performed in order to obtain stable preparations. The measured scattering curves I (Q) were fitted by theoretical models of oblate and prolate ellipsoidal particles.

\section{e) Relaxation spectroscopy; „Poor Man’s“ Temperature Jump (PT-Jump)}

PT-Jump-measurements on DMPC-surfactin solutions were performed at a DMPCconcentration of $2.5 \mathrm{mM}$ and surfactin concentrations of $0.025-0.1 \mathrm{mM}(1-4 \mathrm{~mol} \%)$ in 2.5 
$\mathrm{mM}$ Tricine buffer, $10 \mathrm{mM} \mathrm{NaCl}$; $\mathrm{pH}$ 7.5. Samples were filled into 2 x $10 \mathrm{~mm}$ Quartz cuvettes and thermostatted for 5 min. For 1, 2 and 3 mol\% surfactin the T-jump downwards was performed from 32 to $14{ }^{\circ} \mathrm{C}$ and correspondingly the up-jump from 14 to $32{ }^{0} \mathrm{C}$. For 4 mol\% surfactin the down-jump was from 35 to $24{ }^{\circ} \mathrm{C}$ and the up-jump from 24 to $35^{\circ} \mathrm{C}$, respectively. PT-jump- experiments were started with a down-jump. For these measurements two thermostats were used. With the first one the initial temperature was adjusted, while the second one controlled the temperature in the thermostatable sample holder of our IodineLaser-T-Jump equipment [30] which was used in the time resolved light scattering mode at $350 \mathrm{~nm}$ during the T-jump. Relaxation signals were detected within a time interval of $100 \mathrm{~s}$. In this way the kinetics of processes in the phase transition which proceeded slower than $10 \mathrm{~s}$ could be monitored. The relaxation curves which followed a first-order reaction were recorded using a relaxation detection programme Hi-Tech-Scientific (Hi-Tech, Ltd., Salisburg. UK). Relaxation times $\tau$ ( $\mathrm{s}$ ) and light scattering amplitudes A (a.u.) were obtained by fitting relaxation curves with the evaluation programme Origin. 


\section{Results}

To understand the mechanism of action of the lipoheptapeptide surfactin on biomembranes physicochemical studies of its interaction with DMPC-vesicles were performed which were taken as a model system for a bilayered phospholipid membrane. Surfactin was produced by the recombinant high producer strain B. subtilis OKB 105, as reported by Baumgart et al. [31]. Natural surfactin is a complex mixture of isoforms which show variations both in the length and branching of their fatty acid side chains as well as the amino acid composition and sequence of their peptide moiety. Its isoform composition has been investigated in detail by Kowall et al. [7]. According to matrix-assisted laser desorption/ionization time-of-flight mass spectrometric analysis it contained isoforms with 37\% C13-, 39\% C14- and 24\% C15 ßhydroxy fatty acid components.

\section{1.) Differential scanning calorimetry (DSC) measurements}

The interaction of DMPC-vesicles with surfactin was investigated by differential scanning calorimetry (DSC). In Fig. 2 A-D the effect of surfactin on the phase transition phenomena of DMPC vesicles is demonstrated. The DSC diagram of pure DMPC vesicles shows a single DSC- peak 1 with a maximum at the phase transition temperature $\mathrm{T}_{\mathrm{m} 1}=23.5{ }^{\circ} \mathrm{C}$ (phase transition 1). In the presence of surfactin peak broadening and a shift of $T_{m}$ to lower temperatures was observed. At 4 mol\% surfactin a shoulder appeared at the right flank of the DSC peak which at higher surfactin concentrations in the range of $10-15 \mathrm{~mol} \%$ developed to a separate peak 2 (phase transition 2), while peak 1 was fading out. It completely disappeared at $20 \mathrm{~mol} \%$ surfactin. At $4 \mathrm{~mol} \%$ surfactin $\mathrm{T}_{\mathrm{m} 2}=23{ }^{\circ} \mathrm{C}$ was extrapolated. The two peaks in the

DSC diagram indicate phase transition processes that can be attributed to two forms of DMPC-surfactin aggregates. As is apparent from Table 1, the phase transition temperatures $\mathrm{T}_{\mathrm{m} 1}$ and $\mathrm{T}_{\mathrm{m} 2}$ for both aggregation forms diminished with increasing surfactin concentration. 
This effect was more pronounced for $T_{m 1}$, while $T_{m 2}$ decreased only slightly in the range of surfactin concentrations between 4 and $20 \mathrm{~mol} \%$.

The two peaks in the DSC-curve observed for DMPC-surfactin aggregates were fitted by two Gauss-curves from which the transition enthalpies $\Delta \mathrm{H}_{\text {cal1 }}$ and $\Delta \mathrm{H}_{\mathrm{cal} 2}$ for the phase transition of both forms of DMPC-surfactin aggregates were determined as the areas under these peaks. For peak 1 a small increase of $\Delta \mathrm{H}_{\text {cal1 }}$ from $18.7 \mathrm{~kJ} \mathrm{M}^{-1}$ for pure DMPC-vesicles until approx. $20.6 \mathrm{~kJ} \mathrm{M}^{-1}$ at a surfactin concentration of $4 \mathrm{~mol} \%$ was observed. At higher lipopeptide concentrations $\Delta \mathrm{H}_{\text {cal1 }}$ decreased continuously to values of $1-2 \mathrm{~kJ} \mathrm{M} \mathrm{M}^{-1}$. In contrast $\Delta \mathrm{H}_{\text {cal2 }}$ attributed to phase transition 2 (peak 2) was nearly constant in the range of 5-20 mol\% surfactin.

\section{2.) Investigation of phase transition processes in DMPC-surfactin vesicles by static light}

\section{scattering}

In addition to DSC, the effect of surfactin on the phase transition of DMPC-vesicles was investigated by static light scattering. The scattering intensity I of the DMPC-vesicles around the phase transitions was monitored as a function of temperature in the range of $16-30{ }^{\circ} \mathrm{C}$. The obtained results are shown in Fig. 3A-D. Scanning was started at temperatures above the phase transition point. For pure DMPC-vesicles (Fig. 3A) light scattering intensity increased with decreasing temperature (down scan). S-like scattering profiles were observed showing a strong increase of the intensity at the phase transition and a smaller increase at temperatures below and above the transition. The inflection point corresponds to the phase transition temperature $T_{m 1}$. The phase transition process was reversible, i.e. in the following up scan experiment the initial level of light scattering intensity I was reached at the end temperature of $29{ }^{\circ} \mathrm{C}$, but a small hysteresis effect was observed at temperatures around the phase transition. In this range the scattering intensity detected in the down scan was slightly lower than the 
value obtained in the up scan. Also with increasing surfactin concentrations up to $3 \mathrm{~mol} \%$ the level of light scattering intensity of the DMPC-surfactin-aggregates increased (Fig. 3A) in the down scan experiment. $\mathrm{T}_{\mathrm{m}}$ shifted to lower temperatures (Table 3 ) and the hysteresis effect was getting more and more pronounced (Fig. 3A). At 4 mol\% surfactin an additional second scattering process was observed at temperatures above phase transition 1 indicating a second form of DMPC-surfactin interaction. This result is consistent with the appearance of a second peak in the DSC diagram at this surfactin concentration (phase transition 2). In contrast to the phase transition at $\mathrm{T}_{\mathrm{m} 1}$ in the down scan the light scattering intensity in the region of the phase transition for the second process decreased, when temperature was lowered. Also this phase transition was reversible, but a stronger hysteresis effect was observed than for process 1 . The appearance of two hysteresis effects in the light scattering profile yields further evidence for two forms of DMPC-surfactin-aggregation. With increasing surfactin concentration until 10 mol\% both hysteresis effects diminished and disappeared completely at approx. 15 mol\% (Fig. 3C). Above this concentration the level of light scattering intensity at the starting temperature of the down scan was different from that reached at the end of the following up scan (Fig. 3D).

In Table 2 the data of the influence of surfactin on the transition temperatures $\mathrm{T}_{\mathrm{m} 1}$ and $\mathrm{T}_{\mathrm{m} 2}$ for both forms of DMPC-surfactin aggregates are summarized. In down scan experiments $\mathrm{T}_{\mathrm{m} 1}$ is starting at $23.4{ }^{\circ} \mathrm{C}$ in the case of pure DMPC vesicles decreasing to a value of $19.2{ }^{\circ} \mathrm{C}$ at 10 mol\% surfactin, when the first hysteresis effect was disappearing. The second phase transition started at $4 \mathrm{~mol} \%$ showing a transition temperature $\mathrm{T}_{\mathrm{m} 2}=23.5{ }^{\circ} \mathrm{C}$. These results are in excellent agreement with the DSC data.

\section{3.) Cryo-electron microscopic characterization of DMPC-surfactin-aggregates}

Cryo-electron microscopic measurements have been performed to visualize the DMPC-as 
well as the DMPC-surfactin preparations. Starting from temperatures either above or below the phase transition temperature samples (temperature control was achieved by employing the “controlled environment vitrification system" (CEVS), see Experimental section) were shockfrozen by propelling grids into liquid ethane at cooling rates of $\sim 10^{4} \mathrm{~K} \mathrm{~s}^{-1}$ in order to prevent crystal formation of the solvent water. Electron micrographs of vitrified samples shown in Fig. 4A-D were taken with a Philips Tecnai F20 transmission electron microscope, while maintaining a sample temperature of $89 \mathrm{~K}$. At temperatures higher than $25{ }^{\circ} \mathrm{C}$ pure DMPC preparations showed almost perfect spherical vesicles in a diameter range of 50-100 $\mathrm{nm}$. More detailed inspection of the membrane revealed a tendency to form segments in the order of 50 to $100 \mathrm{~nm}$ The bilayered organisation of the membrane was clearly resolved and gave a thickness of the lipid bilayer in the order of 4-5 $\mathrm{nm}$ which has been determined from sectional profiles according to the method given e.g. by Wang et al. [34].

In the presence of surfactin the overall structure of the vesicles was not significantly affected. However, at surfactin concentrations higher than $4 \mathrm{~mol} \%$ the vesicles were acommpanied by small entities in the order of $50-100 \mathrm{~nm}$, the number of which increased with increasing concentration of the biosurfactant. In most cases these particles display a high contrast bilayer profile identical to that measured for vesicles (Fig. 4C). In almost all cases areas of lower contrast are adjacent to the high contrast bilayer profile, which is a hint for the sheet-like nature of these entities.

Apparently, two kinds of structures, namely unilamellar vesicles (Fig. 4A-C) and sheet-like membrane fragments (Fig. 4C and D), could be visualized by cryo-electron microscopy representing the two aggregation forms detected by DSC- and light scattering measurements. At surfactin concentrations above $15 \mathrm{~mol} \%$ the DMPC-surfactin aggregates have not been resolved by cryo-electron microscopy probably due to size limitations. Therefore, for their structural characterization small angle neutron scattering studies were performed. 


\section{4.) Small angle neutron scattering experiments}

A third form of DMPC-surfactin aggregation was found at higher surfactin concentrations between $15-30 \mathrm{~mol} \%$ of the lipopeptide. In this concentration range the DMPC-surfactin interaction was partially irreversible. This aggregation form was investigated by small angle neutron scattering. In Fig. 5 the scattering intensity I (Q) was measured as a function of the magnitude of the scattering vector $\mathrm{Q}$. The scattering profile was fitted by theoretical model conformations. The best fitting of the experimental data was obtained for two ellipsoidal particles, one of an oblate form of dimensions $\mathrm{R}_{1}=5.1$ and $\mathrm{R}_{3}=1.6 \mathrm{~nm}$ and a second one of a prolate conformation with $\mathrm{R}_{1}=2.9$ and $\mathrm{R}_{3}=7 \mathrm{~nm}$. The structure of DMPC-surfactin micelles has to be clarified in more detail by application of other physicochemical methods.

\section{5.) Poor Man's Temperature (PT)-Jump measurements}

The kinetics of phase transition processes in lipid bilayer membranes can be studied by methods developed for the time resolution of fast chemical reactions, as for example, by temperature-jump measurements. In this work we investigated specifically the slow transients in the main phase transition of DMPC-vesicles and DMPC-surfactin aggregates that were observed in a time scale of 10-100 s applying the PT-jump technique. Measurements were performed at a DMPC-concentration of $2.5 \mathrm{mM}$ in the presence of $0-4 \mathrm{~mol} \%$ of surfactin. Transients in the phase transition were detected by light scattering measurements at a wavelength of $350 \mathrm{~nm}$. Then the sample cuvette equilibrated to the starting temperature was transferred manually to the thermostatted cuvette holder of the our Iodine-Laser-T-Jump equipment [30]. Both down- and up-jumps were performed. The relaxation diagrams, i. e. the change in the scattering intensity $\Delta \mathrm{I}$ as a function of time during the T-jump, were biphasic with a dominating fast part comprising about $90 \%$ of $\Delta \mathrm{I}$ and a minor slower part of approx. $10 \%$. The kinetics of the fast phase could not be resolved, because the corresponding 
relaxation times in the range of micro- and milliseconds are by far lower than the time limit of the PT-instrument of approx. 5 s. However, the slow process could be resolved well. In the down- and up-jump relaxation times of $29.8 \mathrm{~s}$ were determined for pure DMPC-vesicles (see Fig. 6A).

PT-jump experiments performed in the presence of $1 ; 2$ and $4 \mathrm{~mol} \%$ surfactin are shown in Figs. 6B-D. As is apparent from Table 3, surfactin accelerates the slow process observed in the phase transition of DMPC-vesicles. At a lipopeptide concentration of 4 mol $\% \tau$ was decreased to a value of $18.5 \mathrm{~s}$. At higher surfactin concentrations, when the DMPC-vesicles were completely converted into sheet-like lamellar membrane fragments, the slow phase in the relaxation diagram disappeared. 


\section{Discussion}

Surfactin, a lipopeptide biosurfactant from Bacillus subtilis, diplays a characteristic horsesaddle topology [6,35]. It is an attractive natural compound showing a broad spectrum of antimicrobial [2,10,15] and antiviral [16-19] activities. Therefore, it is of great medical and biotechnological interest. The action of surfactin on microorganisms and viruses mainly depends on its membrane-active properties $[15,18]$. Because of its amphiphilic structure it is able to efficiently penetrate into biomembranes, ultimately leading to their disintegration $[15,18]$. Therefore, studies on the surfactin-biomembrane interaction are an indispensible prerequisite to understand its biological action. With this aim we investigated the effects of surfactin on DMPC vesicles as a model of a phospholipid bilayer membrane by physicochemical techniques. Differential scanning calorimetry (DSC) and static light scattering as well as methods for the structural characterization of lipid-surfactin aggregates, as cryo-electron microscopy and small angle neutron scattering (SANS) were applied.

The results obtained by DSC- and light scattering experiments are in excellent agreement. Both techniques give evidence for two forms of DMPC-surfactin aggregates. DSCthermograms show two phase transition peaks, while light scattering profiles indicate two branches with pronounced hysteresis phenomena. Nearly the same values of the transition temperatures $\mathrm{T}_{\mathrm{m} 1}$ and $\mathrm{T}_{\mathrm{m} 2}$ of $23-23.5{ }^{\circ} \mathrm{C}$ have been determined by both methods. With increasing surfactin concentrations DSC-measurements show a progressive broadening of the phase transition peaks and a shift of $T_{\mathrm{m} 1}$ and $\mathrm{T}_{\mathrm{m} 2}$ to lower values indicating a decrease in the strength and cooperativity of the lipid-lipid and lipid-surfactin interaction in the bilayer membrane. The second peak in the thermogram and the second branch of the light scattering profiles appeared at surfactin concentrations above $4 \mathrm{~mol} \% . \mathrm{T}_{\mathrm{m} 2}$ showed a smaller decrease with increasing surfactin concentration than $\mathrm{T}_{\mathrm{m} 1}$. Both aggregation forms revealed characteristic differences in the dependence of $\Delta \mathrm{H}_{\mathrm{cal}}$ on the surfactin concentration. For $\Delta \mathrm{H}_{\mathrm{cal}}$ 
an initial increase was observed until 4 mol\% followed by a pronounced decrease until approximately $15 \mathrm{~mol} \%$, while $\Delta \mathrm{H}_{\text {cal } 2}$ for the phase transition of the second form remained nearly constant between 4 and $30 \mathrm{~mol} \%$ surfactin. At surfactin concentrations higher than 20 mol\% irreversible effects were observed. Under these conditions the original level of light scattering at the start of the down scan was not reached again at the end of the opposite upscan. At $30 \mathrm{~mol} \%$ surfactin in the up-scan experiment no change in the scattering intensity occured, i. e. the DMPC-surfactin system was residing in the state reached after the down scan. These effects indicate a third form of DMPC-surfactin aggregates.

Cryo-electron microscopic and small angle neutron scattering studies proved the existence of the three aggregation forms indicated by DSC and light scattering experiments. Particularly cryo-electron micrographs illustrate aggregation forms 1 and 2 (Fig. 4A-D). Pure DMPC-gave vesicles with a clearly resolved bilayer profile. Additionally, a segmentation of the membrane in the order of $50-100 \mathrm{~nm}$ can be seen. This effect is probably due to different packing density between the outer and inner membrane layer which has been proven in small unilamellar vesicles of DMPC by NMR [36] and was later refered to be due to electrostatic effects [37]. At surfactin concentrations higher than $4 \mathrm{~mol} \%$ the number of the vesicular particles decreased. They were progressively accompanied by smaller objects with a length of $50-100 \mathrm{~nm}$, which partially provided a bilayer profile similar to that observed in the vesicles. Although at first sight the high contrast of this bilayer profile suggests the fragments to show a rod-like molecular architecture, a closer examination indicated that the structure of the fragments must be understood of being organized by more or less planar bilayered membrane sheets. Since the objects have been trapped in the layer of the solvent upon vitrification in different spatial orientation with respect to the incident electron beam, differences in shape and structure depending on the view angle were revealed. Arrows in Fig. 4C and D clearly point to tilted views of such membrane fragments displaying the sheet-like topview 
characteristics with the bilayered side-view profile. In Fig. 4C, however, notably the topview projection image reveals some regular striations indicating additional undulations of the membrane fragments (encircled) typically observed for highly flexible membranes, which is also clearly visble in a more tilted object in Fig. 4C, indicated by the dashed arrow. Surfactin, therefore, is thought to act like a scissor shearing off smaller membrane areas from the vesicle surface, presumably along the vesicle segmentation "crestlines". Such flexible membrane fragments were observed in different orientation by cryo-TEM.

At surfactin concentrations higher than $20 \mathrm{~mol} \%$, at which irreversible effects have been observed by DSC and light scattering measurements, small particles appeared which could not properly be resolved by cryo-electron microscopy. Therefore, their size and conformation were investigated by small angle neutron scattering. The scattering profiles were fitted well by two models which cannot yet be discriminated. These particles presumably represent mixed micelles of DMPC and surfactin of ellipsoidal form. This process was accompanied by a strong decrease in light scattering.

Similar phenomena have also been reported for the solubilization of lipid bilayers by synthetic surfactants which are accompanied by morphological changes of the bilayer and the emergence of mixed micelles [38]. As is the case for the interaction of surfactin with DMPC lipid vesicles sometimes intermediates in form of disks or sheet-like bilayer fragments occurred. By incorporation of the biosurfactant into the DMPC-bilayer intermediate sheet-like lamellar fragments start to form that coexist with the vesicles, until they are completely disintegrated. At higher surfactin concentrations finally ellipsoidal mixed micelles are formed.

Preliminary DSC and light scattering studies on the interaction of surfactin with L- $\alpha$ dipalmitoyl phosphaditylcholine (DPPC) have been performed. Similar phenomena have been observed as for DMPC vesicles, but the effects appeared at appreciably higher surfactin 
concentrations (data not shown). Also here the thermograms and the light scattering profiles indicate two forms of DPPC-surfactin aggregates. There is also a progressive decrease of the phase transition temperature and a broadening of the DSC- peaks with increasing surfactin concentration. In contrast to DMPC, however, the second DSC-peak arising at 5-10 mol\% surfactin was found at lower temperatures than the peak for the vesicular form. Both the shifts of $\mathrm{T}_{\mathrm{m} 1}$ and $\Delta \mathrm{Hcal}_{1}$ at increasing surfactin concentration were smaller than in the case of the DMPC-surfactin system. These features imply a weaker interaction of surfactin with DPPC than with DMPC which can mainly be explained by the different lengths of their fatty acid side chains. DMPC has a C14-side chain which matches the length of a major portion of the ß-hydroxy fatty acid components of natural surfactin. This seems more favorable in the interaction with the myristoyl-acyl chains of DMPC, while the molecular forces with the C16palmitoyl-side chains of DPPC are weaker. Similar results had previously been reported by Grau et al. [25].

Also the light scattering profiles observed for the phase transitions of DMPC and DPPC were qualitatively similar showing two branches with characteristic hysteresis phenomena. For the vesicular form of the DPPC-surfactin aggregates observed until a surfactin concentration of approx. $5 \mathrm{~mol} \%$ again the scattering intensity increased in the down-scan experiment, while the opposite behaviour was found above this concentration. As for DMPC irreversible lipidbiosurfactant interaction ocurred at surfactin concentration higher than $20 \mathrm{~mol} \%$ which finally resulted in mixed micellar aggregates of low light scattering intensities.

Kinetic studies on the main phase transition of unilamellar lipid vesicles by iodine laser temperature jump experiments in combination with time resolved cryo-electron microscopy were performed by Groll et al. [30] for DMPC vesicles. These authors developed a model for the hierarchie of the dynamic processes occuring during the phase transition from the crystalline to the fluid state, as illustrated in Fig. 7. A sequence of at least five events could be 
resolved. The initial fast relaxation processes observed in the nano- to microsecond range were attributed to kink formation of the hydrocarbon chains followed by head group rotation. Rotational isomers of DMPC are formed by conversion of the all trans- into the cis -trans conformation increasing their lateral mobility. In the phase transition starting from the crystalline hexagonal array intermediate lipid clusters/ domains are formed which arise in the micro- to millisecond time scale followed by a slow conversion of the lipid molecules into the fluid state. This process has not yet been explored in detail. According to the model of Groll et el. [30] the supramolecular intermediates appear as patches segregated within the bilayer. They can be made visible by time-resolved cryo-electron microscopy. The detection and kinetic analysis of these unknown slow processes was another aim of this paper. For this purpose the „Poor Man's“ temperature jump technique was used by which transients in the range of seconds and the influence of surfactin on these slow relaxation processes could efficiently be investigated. They were only observed in the case of pure DMPC-vesicles and vesicular DMPC-surfactin aggregates at low surfactin concentrations until 4 mol\%. At higher lipopeptide concentrations, when the vesicles were disintegrated into lamellar membrane fragment sheets, the slow phase in the relaxation diagram completely disappeared.

Taking together the results of our thermodynamic, kinetic and structural studies the following features of the DMPC-surfactin interaction can be proposed: In all experiments the surfactin concentration in solution was higher than the critical micelle concentration CMC of approximately $10 \mu \mathrm{M}[13,28]$. Therefore, it has to be assumed that always assemblies of the lipopeptide were taken up into the DMPC-lipid membrane. Our thermodynamic and kinetic data imply that at surfactin concentrations lower than 4 mol\% lipopeptide molecules are dispersed within the bilayer membrane and interact with the adjacent lipid molecules. Because of the bulky polar head part of surfactin the hexagonal array of the lipid bilayer is gradually destabilized increasing the fluidity of hydrocarbon side chains of the lipid 
molecules. Under these conditions an increase of $\Delta \mathrm{H}_{\text {call }}$, the appearance of a slow phase in the relaxation diagrams of the PM-T-jump experiments and a decrease of the relaxation times with increasing surfactin were observed. This interpretation is in agreement with mono- and bilayer studies $[9,25]$ which have shown that surfactin is miscible with phospholipids at low lipopeptide concentration. Increasing the surfactin concentration above $4 \mathrm{~mol} \%$ patches of surfactin clusters/domains persist ultimately giving rise to a disruption of the DMPC vesicles into lamellar fragment sheets. In this state surfactin is almost immiscible within the leaflet. This view is consistent with the model of Deleu et al. [26,27] mainly derived from atomic force microscopy. Under these conditions $\Delta \mathrm{H}_{\text {call }}$ strongly decreased and the slow phase of the relaxation profiles disappeared. $\Delta \mathrm{H}_{\text {cal2 }}$ characteristic for the phase transition of the lamellar DMPC-surfactin sheet-like lamellar fragments was nearly independent of the surfactin concentration indicating only weak interactions between lipid and surfactin molecules. According to the data reported by Grau et al. [25] the appearance of the second peak in the DSC-thermogram can be attributed to a lateral phase segregation of surfactin-rich domains in agreement with their fluid-phase immiscibility. Presumably surfactin integrates into the lamellar membrane fragments by growth of the biosurfactant clusters rather than by direct interaction with the lipid molecules. There is a coexistence between DMPC-rich and surfactin-rich domains, as demonstrated by Grau et al. [25] and Deleu et al. [26,27]. Finally, at surfactin concentrations higher than $15 \mathrm{~mol} \%$ conversion of the membrane fragments into smaller DMPC-surfactin mixed micelles of ellipsoidal conformation occured which were characterized by small angle neutron scattering experiments. Consistent with the phenomena observed for the interaction of surfactin with phospholipid model membranes of DMPC and DPPC the biological action of surfactin on mycoplasma [15] and enveloped viruses [18] is exerted by its micellar rather than by its monomolecular form. Below the critical micelle concentration only small inactivation effects were observed. 
Our studies on the influence of surfactin on the DMPC-model membrane are a contribution to understand a) the effects of surfactin and related lipopeptides on biomembranes and whole cells as well as b) the manifold biological activities of this biosurfactant on the molecular level. 


\section{Conclusions}

In this paper three forms of DMPC-surfactin aggregates have been detected by differential scanning calorimetry and light scattering. They were structurally characterized by cryoelectron microscopy and small angle neutron scattering. The solubilization of DMPC-vesicles by surfactin showed a typical three-stage sequence.

1.) At low surfactin concentration until approximately $4 \mathrm{~mol} \% \mathrm{DSC}$ and light scattering showed nearly the same transition temperatures $\mathrm{T}_{\mathrm{m} 1}$ of $22-23.5{ }^{\circ} \mathrm{C}$ and an increasing $\Delta \mathrm{H}_{\text {call }}$. Cryo-electron micrographs exhibited vesicular particles, some of them showing a partioning of their lipid envelope. Under these conditions PM-T-jump experiments revealed a specific slow phase in the relaxation diagram in the time scale of 20-30 s. Surfactin accelerated this relaxation process which disappeared at $4 \mathrm{~mol} \%$.

2.) Between $4-15 \mathrm{~mol} \%$ surfactin the DMPC vesicles were progressively disrupted by the biosurfactant into sheet-like lamellar membrane fragments with a length of 50-100 nm and a width of 4-5 $\mathrm{nm}$, as visualized by cryo-electron microscopy. In this case $\mathrm{T}_{\mathrm{m} 2}$ decreased only slightly. $\Delta \mathrm{H}_{\mathrm{cal} 2}$ remained nearly constant. Under these conditions lipid-rich and surfactin-rich domains presumably alternate with each other within the bilayer membrane. In this state surfactin is nearly immiscible in the DMPC-membrane and the interaction between lipid and biosurfactant is weak, as indicated by the low value of $\Delta \mathrm{H}_{\mathrm{cal2}}$. Here the slow transients in the PT-jump experiments disappeared.

3.) At surfactin concentrations above $15 \mathrm{~mol} \%$ smaller, ellipsoidal particles were formed in a partially irreversible process which presumably represent mixed micelles of DMPC and surfactin. Their size and conformation was determined by small angle neutron scattering fitting the scattering profile by model conformations. 


\section{References}

[1] K. Arima, A. Kakinuma and G.Tamura, Surfactin, a crystalline peptide lipid surfactant produced by Bacillus subtilis: Isolation, characterization and its inhibition of clot formation, Biochem. Biophys. Res. Commun. 31 (1968) 488-494.

[2] J. Vater, Lipopeptides, an attractive class of microbial surfactants, Progr. Colloid Polymer Sci. 72 (1986) 12-18.

[3] A. Kakinuma, M. Hori, M. Isono, G. Tamura and K. Arima, Determination of amino acid sequence in surfactin, a crystalline peptidelipid surfactant produced by Bacillus subtilis, Agr. Biol. Chem. 33 (1969) 971-972.

[4] A. Kakinuma, H. Sugino, M. Isono, G. Tamura and K. Arima, Determination of fatty acid in surfactin and elucidation of the structure of surfactin, Agr. Biol. Chem. 33 (1969) 973-976.

[5] A. Kakinuma, M. Hori, H. Sugino, J. Yoshida, M. Isono, G. Tamura and K. Arima, Determination of the location of lactone ring in surfactin, Agr. Biol. Chem. 33 (1969) 1523-1524.

[6] J.-M. Bonmatin, M. Genest, H. Labbé and M. Ptak, Solution three-dimensional structure of surfactin: A cyclic lipopeptide studied by ${ }^{1} \mathrm{H}-\mathrm{NMR}$, distance geometry, and molecular dynamics, Biopolymers 34 (1994) 975-986.

[7] M. Kowall, J. Vater, B. Kluge, T. Stein, P. Franke and D. Ziessow, Separation and characterization of surfactin isoforms produced by Bacillus subtilis OKB 105, J. Colloid Interface Sci. 204 (1998) 1-8.

[8] R. Maget-Dana and M. Ptak, Interfacial properties of surfactin, J. Colloid Interface Sci.153 (1992) 285-291.

[9] R. Maget-Dana and M. Ptak, Interactions of surfactin with membrane models, Biophys. J. 68 (1995) 1037-1943. 
[10] A.W. Bernheimer and L.S. Avigad, Nature and properties of a cytolytic agent produced by Bacillus subtilis, J. Gen. Microbiol. 61 (1970) 361-369.

[11] H. Razafindralambo, M. Paquot, A. Baniel, Y. Popineau, C. Hbid, P. Jacques and P. Thonart, Foaming properties of surfactin, a lipopeptide biosurfactant from Bacillus subtilis, J. Am. Oil Chem. Soc. 73 (1996) 149-151.

[12] M. Deleu, H. Razafindralambo, Y. Popineau, P. Jacques, P. Thonart and M. Paquot, Interfacial and emulsifying properties of lipopeptides from Bacillus subtilis, Colloids and Surfaces, A. Physicochem. Engineer. Aspects 152 (1999) 3-10.

[13] Y. Ishigami, M. Osman, H. Nakahara, Y. Sano, R. Ishiguro and M. Matsumoto, Significance of $\beta$-sheet formation for micellization and surface adsorption of surfactin, Colloids Surfaces B: Biointerfaces 4 (1995) 341-348.

[14] A. Knoblich, M. Matsumoto, R. Ishiguro, K. Murata, Y. Fujiyoshi, Y. Ishigami and M. Osman, Electron cryo-microscopic studies on micellar shape and size of surfactin, an anionic lipopeptide, Colloids Surfaces B: Biointerfaces 5 (1995) 43-48.

[15] D. Vollenbroich, G. Pauli, M. Özel and J. Vater, Antimycoplasma properties and application in cell culture of surfactin, a lipopeptide antibiotic from Bacillus subtilis, Appl. Environ. Microbiol. 63 (1997) 44-49.

[16] N. Naruse, O. Temyo, S. Kobaru, H. Kamei, T. Miyaki, M. Konishi and T. Oki, Pumilacidin, a complex of new antiviral antibiotics- production, isolation, chemical properties and biological activity, J. Antibiot. (Tokyo) 43 (1990) 267- 280.

[17] H. Itokawa, T. Miyashita, H. Morita, K. Takeya, T. Hirano, M. Homma and K. Oka, Structural and conformational studies of $\left[\mathrm{Ile}^{7}\right]$ and $\left[\mathrm{Leu}^{7}\right]$ surfactins from Bacillus subtilis natto, Chem. Pharm. Bull. 42 (1994) 604-607.

[18] D. Vollenbroich, M. Özel, J. Vater, R.M. Kamp and G. Pauli, Mechanism of 
inactivation of enveloped viruses by the biosurfactant surfactin from Bacillus subtilis, Biologicals 25 (1997) 289-297.

[19] M. Kracht, H. Rokos, M. Özel, M. Kowall, G. Pauli and J. Vater, Antiviral and hemolytic activities of surfactin isoforms and their methylester derivatives, J. Antibiot. (Tokyo) 52 (1999) 613-619.

[20] Y. Kameda, S. Ouhira, K. Matsui, S. Kanamoto, T. Hase and T. Atsusaka, Antitumor activity of Bacillus natto V. Isolation and characterization of surfactin in culture medium of Bacillus natto KMD 2311, Chem. Pharm. Bull. 22 (1974) 938-944.

[21] Y. Imai, H. Sugino, T. Fujita and A. Kakinuma, Hypocholesterolemic effect of surfactin, a novel bacterial peptidelipid, J. Takeda Res. Lab. 30 (1971) 728-734.

[22] L.Thimon, F. Peypoux and G. Michel, Interactions of surfactin, a biosurfactant from Bacillus subtilis, with inorganic cations, Biotechnol. Lett. 14 (1992) 713-718.

[23] D. Sheppard, C. Jumarie, D.G. Cooper and R. Laprade, Ionic channels induced by surfactin in planar bilayer membranes, Biochim. Biophys. Acta 1064 (1991) 13-23.

[24] L. Thimon, F. Peypoux, J. Wallach and G. Michel, Ionophorous and sequestering properties of surfactin, a biosurfactant from Bacillus subtilis, Colloids Surfaces B: Biointerfaces 1 (1993) 57-62.

[25] J.C. Grau, F. Gómez Fernández, F. Peypoux and A. Ortiz, A study on the interaction of surfactin with phospholipid vesicles, Biochim. Biophy. Acta 1418 (1999) 307-319.

[26] M. Deleu, M. Paquot, P. Jacques, P. Thonart, Y. Adriaensen and Y.F. Dufrene, Nanometer scale organization of mixed surfactin/phophatidylcholine monolayers, Biophys. J. 77 (1999) 2304-2310.

[27] M. Deleu, K. Nott, R. Brasseur, P. Jacques, P. Thonart and Y.F. Dufrene, Imaging mixed lipid monolayers by dynamic atomic force microscopy, Biochim. Biophys. Acta 1513 (2001) 55-62. 
[28] H. Heerklotz and J. Seelig, Detergent-like action of the antibiotic peptide surfactin on lipid membranes, Biophys. J. 81 (2001) 1547-1554.

[29] C. Carrillo, J.A. Teruel, F.J. Aranda and A. Ortiz, Molecular mechanism of membrane permeabilization by the peptide antibiotic surfactin, Biochim. Biophys. Acta 1611 (2003) 91-97.

[30] R. Groll, A. Böttcher, J. Jäger and J.F. Holzwarth, Temperature dependent intermediate structures during the main phase transition of dimyristoylphosphatidylcholine vesicles, a combined iodine laser temperature jump and time resolved cryoelectron microscopic study, Biophys. Chem. 58 (1996) 53-65.

[31] F. Baumgart, B. Kluge, C. Ullrich, J. Vater and D. Ziessow, Identification of amino acid substitutions in the lipopeptide surfactin using 2D NMR spectroscopy, Biochem. Biophys. Res. Commun. 177 (1991) 998-1005.

[32] Bellare, J.R., Davis, H.T. , Scriven, L.E. and Talmon, Y. Controlled environment vitrification system: an improved sample preparation technique. J. Electron Microsc. Tech. 10 (1988) 87-111.

[33] J. Penfold, E. Staples, I. Tucker, G.J.T. Tiddy and A. Khan Lodi, Shear-induced structures in concentrated surfactant micellar phases, J. Appl. Cryst. 30 (1997) 744749.

[34] L. Wang, P.S. Bose and J. Sigworth, Using cryo-EM to measure the dipole potential of a lipid membrane, Proc. Natl. Acad. Sci. USA 103 (2006) 18528-18533.

[35] P. Tsan, L. Volpon, F. Besson and J.-M. Lancelin, Structure and dynamics of surfactin studied by NMR in micellar media, J. Am. Chem. Soc. 129 (2007) 19681977.

[36] A.C. McLaughlin, F. Podo and J.K. Blaise, Temperature and frequency dependence of 
longitudinal proton relaxation times in sonicated lecithin dispersions, Biochim. Biophys.Acta 330 (1973) 109-121.

[37] R. Lawaczek, M. Kainosho and S.I. Chan, The formation and annealing of structural defects in bilayer vesicles, Biochim. Biophys. Acta 443 (1976) 313-330.

[38] M. Almgren, Mixed micelles and other structures in the solubilization of bilayer lipid membranes by surfactants, Biochim. Biophys. Acta 1508 (2000) 146-163. 


\section{Legends}

Fig.1 Structure of surfactin

Fig. 2

Molar specific heat $\mathrm{c}_{\mathrm{p}}{ }^{\mathrm{m}}$ of DMPC-vesicles as a function of temperature determined by differential scanning calorimetry at various surfactin concentrations.

Thermograms were measured at following surfactin concentrations: A) 0, 1, 2 and $3 \mathrm{~mol} \%$; B) 4, 5 and $6 \mathrm{~mol} \%$; C) 7, 8 and $10 \mathrm{~mol} \%$; D) 15,20 and $30 \mathrm{~mol} \%$. Scan rate: $0.5{ }^{\circ} \mathrm{C} \mathrm{min}^{-1}$.

Fig. 3

Light scattering intensity at $\lambda=300 \mathrm{~nm}$ of DMPC-vesicles as a function of temperature at various surfactin concentrations in down $\operatorname{scan}(\nabla, \circ, \diamond)$ and up $\operatorname{scan}(\boldsymbol{\Lambda}, \bullet, \bullet)$ experiments. Light scattering profiles were measured at following surfactin concentrations: A) $0(\nabla, \mathbf{\Lambda})$, $2(\circ, \bullet)$ and $3(\diamond, \bullet) \mathrm{mol} \% ; \mathrm{B}) 7(\nabla, \mathbf{\Delta}) \mathrm{mol} \% ; \mathrm{C}) 15(\diamond, \bullet) \mathrm{mol} \%$ and D) $20(\diamond, \bullet)$ and $30(\nabla, \mathbf{\Delta})$ $\mathrm{mol} \%$.

Fig. 4

Cryo-electron micrographs of pure DMPC (A) and DMPC-surfactin (B-D) preparations. The DMPC concentration in all cases was $2.5 \mathrm{mM}$. The surfactin concentration was 2 (B), 4 (C) and 8 (D) mol \%, respectively. Arrows in (A) label a typical example of vesicle membrane segmentation. Arrows in (C) and (D) indicate membrane fragments in different tilted spatial orientations. The sheet-like organisation as well as the bilayer profile is clearly visible. The encircled area in (C) points to an undulated membrane sheet in top-view orientation. The 
dashed arrow in (D) points to an entity providing all characteristics at the same time: sheet character with undulations as well as bilayer profile.

Fig. 5

Small angle neutron scattering intensity I (Q) as a function of the scattering vector Q of DMPC-vesicles in the presence of $30 \mathrm{~mol} \%$ surfactin.

The DMPC concentration was $2.5 \mathrm{mM}$. The lipid was dissolved in $2.5 \mathrm{mM}$ Tricine/ $\mathrm{D}_{2} \mathrm{O}$ buffer, 10 mM NaCl; pH 7.5.

Fig. 6

Poor Man's temperature -jump experiment of DMPC vesicles in the presence of various surfactin concentrations.

The amplitudes of light scattering were detected at $\lambda=350 \mathrm{~nm}$ during the PT-jump as a function of time in the down- and up-jump.

Pure DMPC-vesicles (A); DMPC-surfactin aggregates at surfactin concentrations of 1 (B), 2 (C) and 4 (D) mol\%. Scattering angle: $90^{\circ}$.

Fig. 7

Model of the main phase transition of unilamellar lipid vesicles deduced from T-jump experiments in combination with time resolved cryo-electron microscopy. 

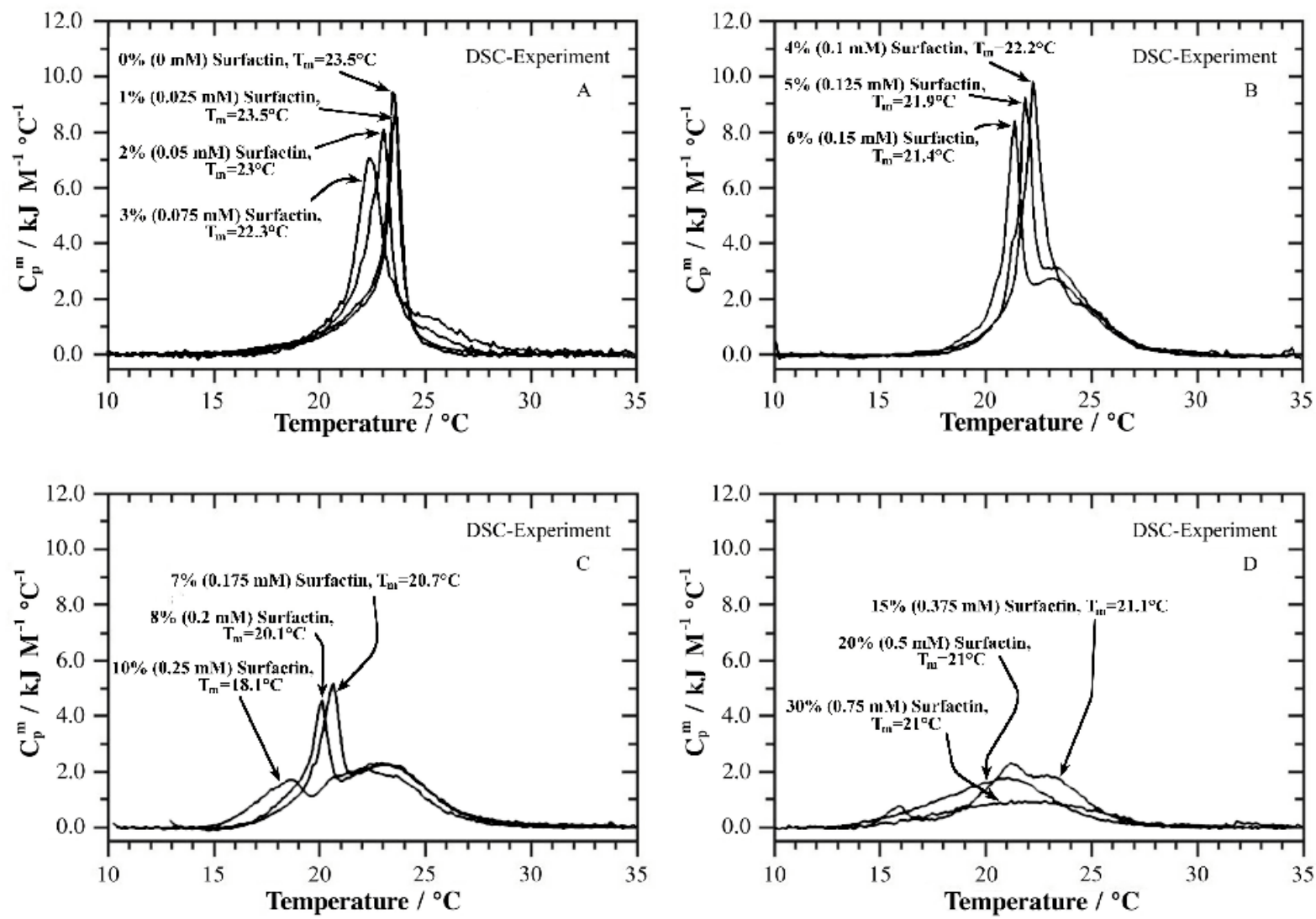

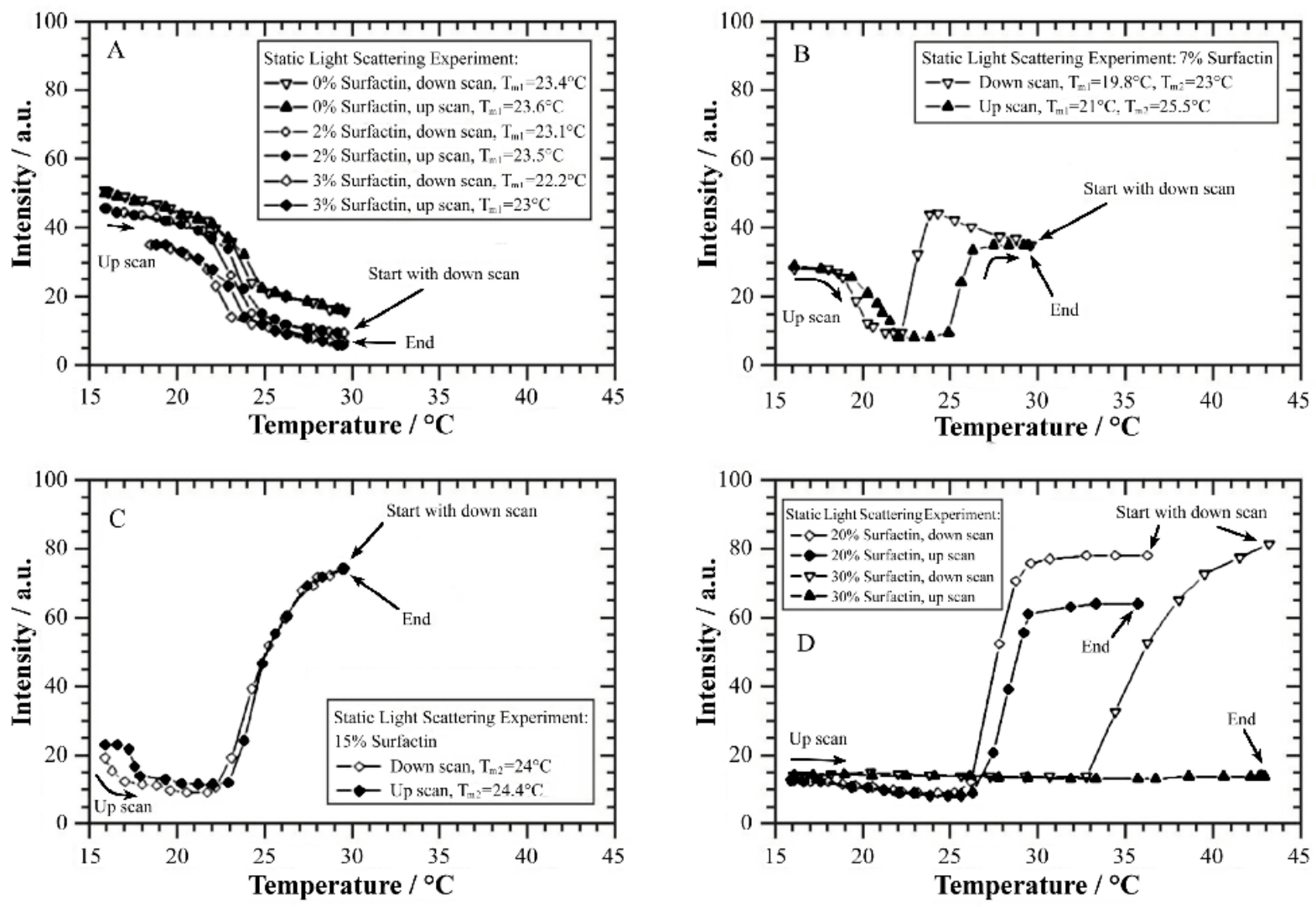
ACCEPTED MANUSCRIPT
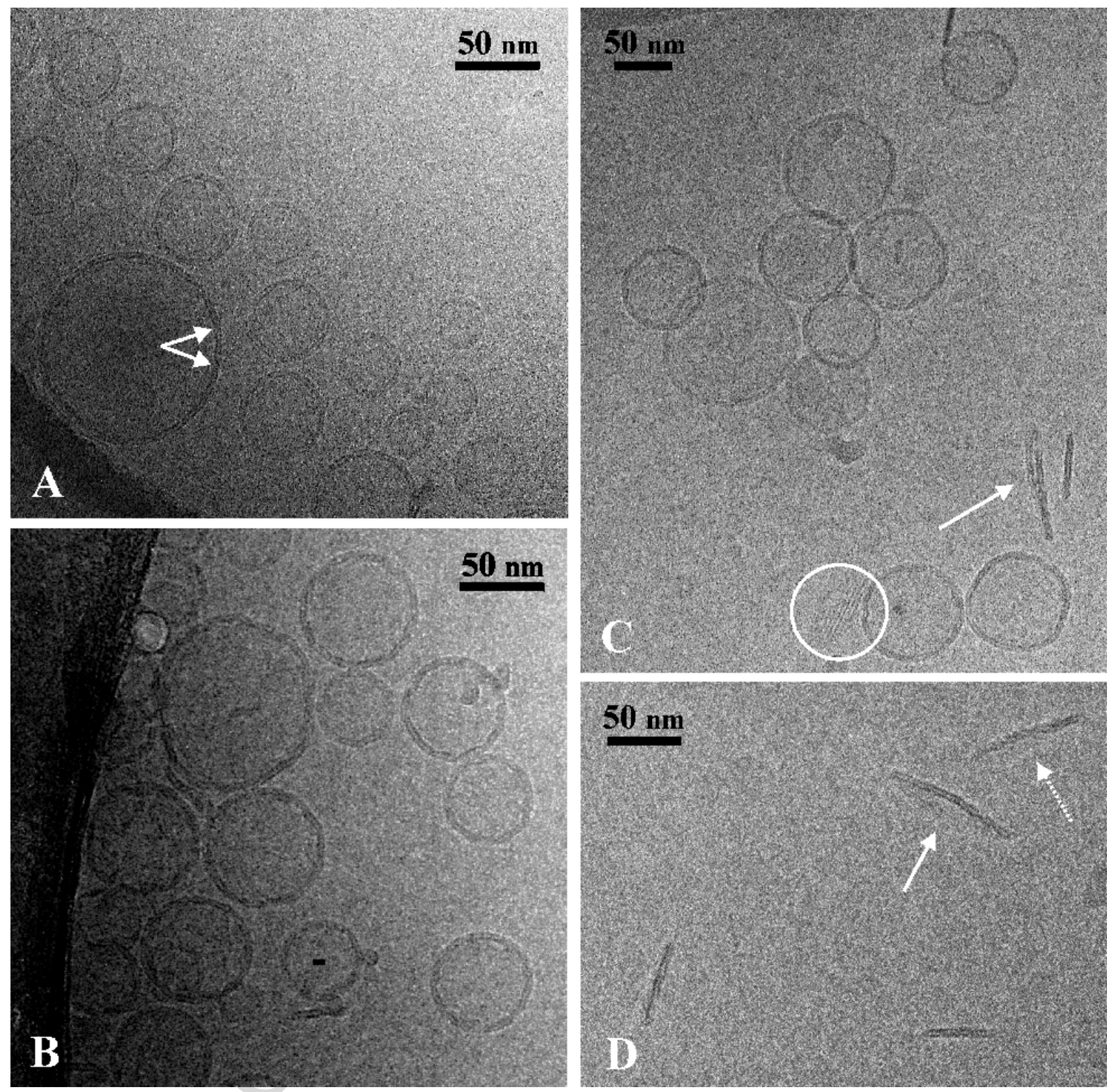
ACCEPTED MANUSCRIPT

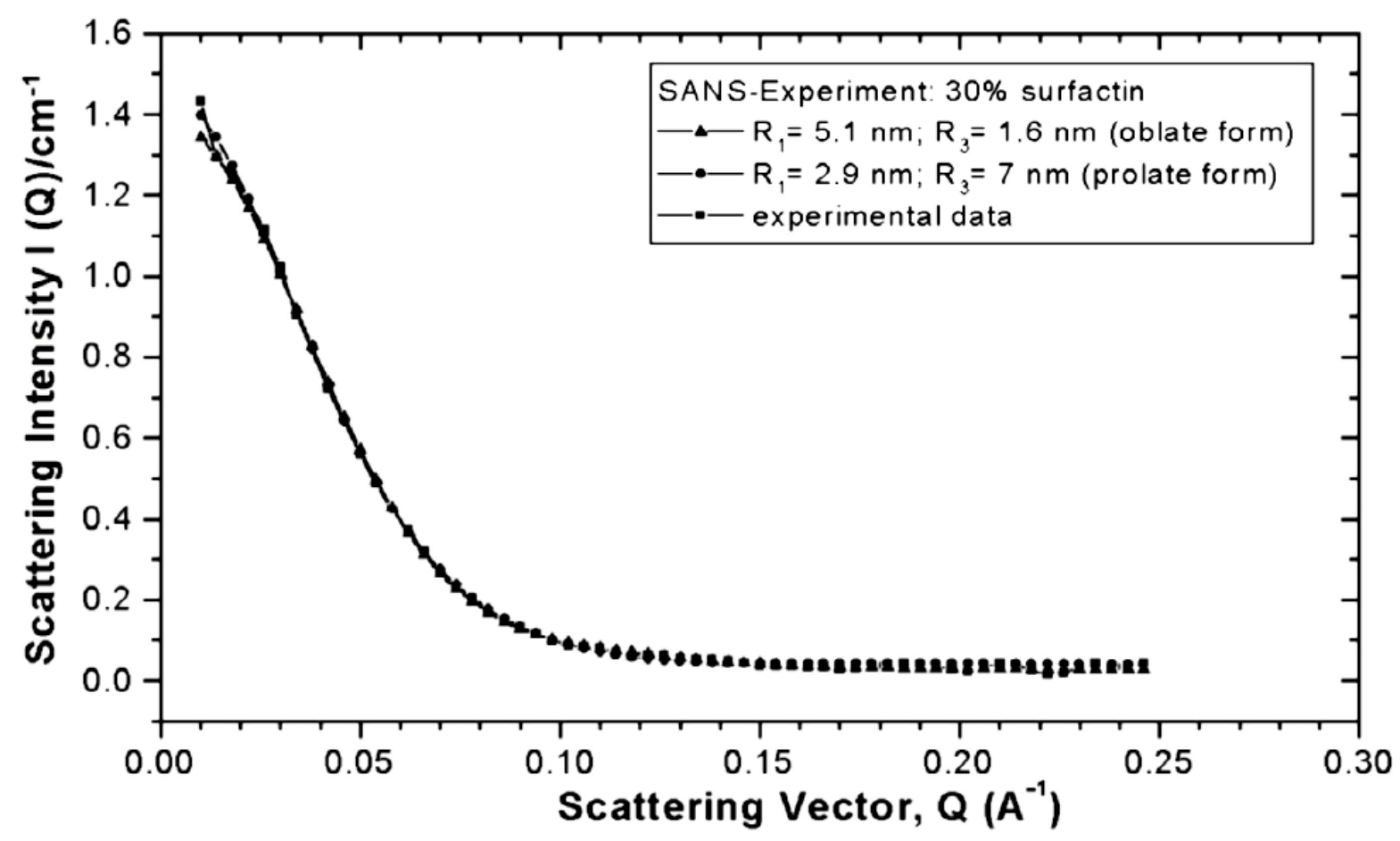



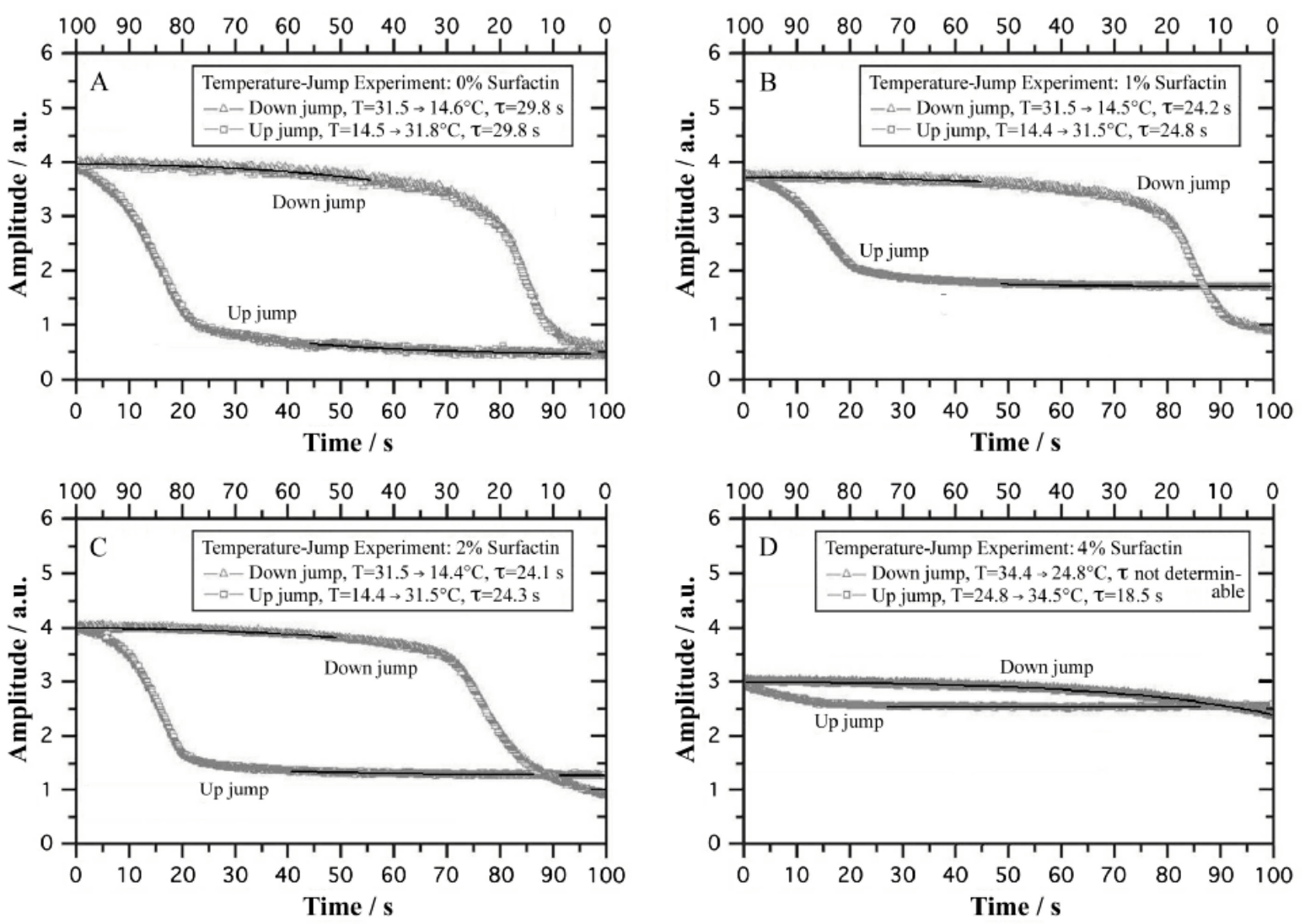
(1)

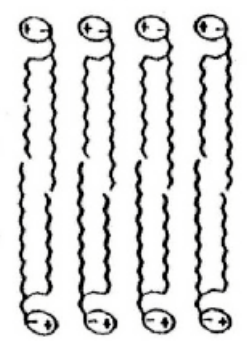

(cross-section)

(7)

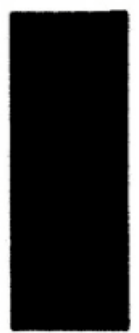

temp. $>$ transition temp. equilibrium $\leqslant$ second tèmp. $\ll$ transition temp.

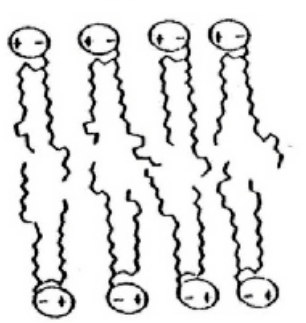
crystalline

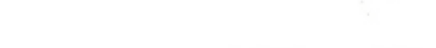

$$
\text { fluid }
$$

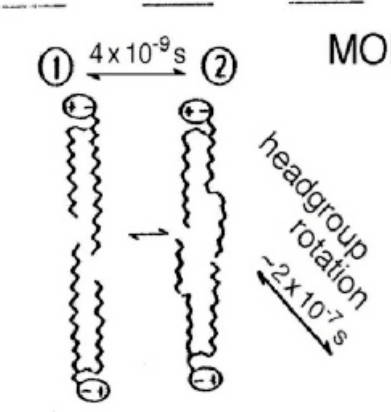

cross-section

$\oplus$ headgroups

m nydrocarbon chains

\section{nanoseconds to seconds}
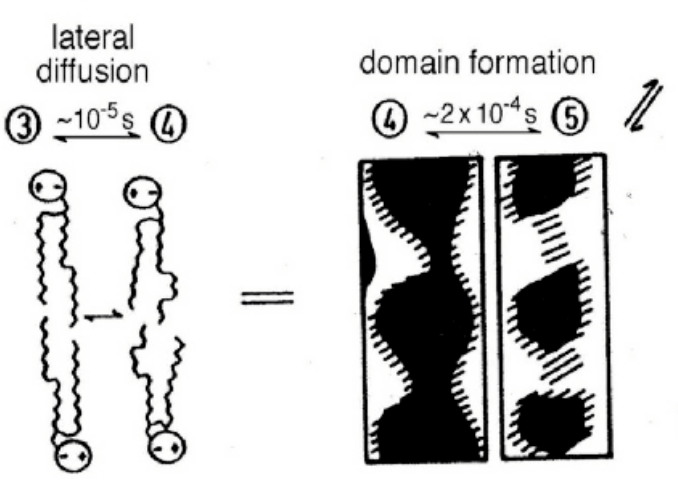

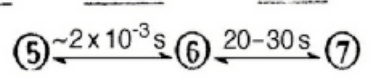

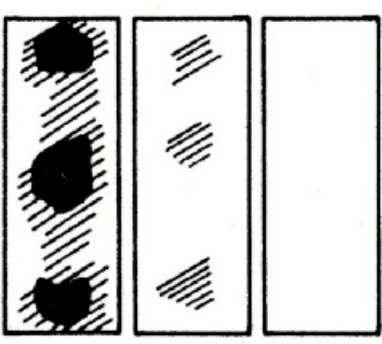

top-plan-view

- cluster of crystalline 位termediate lipids

O fluid lipids 
Table 1. The effect of surfactin on $\mathrm{T}_{\mathrm{m}}$ and $\Delta \mathrm{H}_{\mathrm{cal}}$ of the endothermic phase transition of DMPC-vesicles determined by differential scanning calorimetry (DSC)

\begin{tabular}{|c|c|c|c|c|c|c|}
\hline \multicolumn{2}{|c|}{ Surfactin concentration } & \multirow{2}{*}{$\begin{array}{l}\mathrm{T}_{\mathrm{m} 1} \\
{ }^{\circ} \mathrm{C}\end{array}$} & \multirow{2}{*}{$\begin{array}{l}\mathrm{T}_{\mathrm{m} 2} \\
{ }^{\circ} \mathrm{C}\end{array}$} & \multirow{2}{*}{$\begin{array}{c}\Delta \mathrm{H}_{\text {cal total }} \\
\mathrm{kJ} / \mathrm{M}\end{array}$} & \multirow{2}{*}{$\begin{array}{c}\Delta \mathrm{H}_{\mathrm{call}} \\
\mathrm{kJ} / \mathrm{M}\end{array}$} & \multirow{2}{*}{$\begin{array}{l}\Delta \mathrm{H}_{\mathrm{cal} 2} \\
\mathrm{~kJ} / \mathrm{M}\end{array}$} \\
\hline $\mathrm{mM}$ & $\mathrm{mol} \%$ & & & & & \\
\hline 0.000 & 0 & $23.5 \pm 0.0$ & & $18.7 \pm 0.3$ & $18.7 \pm 0.3$ & \\
\hline 0.025 & 1 & $23.5 \pm 0.1$ & & $18.7 \pm 0.3$ & $18.7 \pm 0.3$ & \\
\hline 0.050 & 2 & $23.0 \pm 0.1$ & & $19.7 \pm 0.2$ & $19.7 \pm 0.2$ & \\
\hline 0.075 & 3 & $22.3 \pm 0.1$ & & \pm 0.3 & $20.1 \pm 0.3$ & \\
\hline 0.100 & 4 & $22.2 \pm 0.0$ & $23.0 \pm 0.1$ & $20.6 \pm 0.3$ & $13.7 \pm 0.2$ & $7.1 \pm 0.1$ \\
\hline 0.125 & 5 & $21.9 \pm 0.2$ & $23.0 \pm 0.1$ & $20.4 \pm 0.7$ & $10.9 \pm 0.4$ & $11.5 \pm 0.4$ \\
\hline 0.150 & 6 & $21.4 \pm 0.1$ & $22.7 \pm 0.1$ & $19.4 \pm 1.1$ & $9.6 \pm 0.6$ & $11.8 \pm 0.7$ \\
\hline 0.175 & 7 & $20.7 \pm 0.2$ & $22.5 \pm 0.1$ & $19.3 \pm 1.1$ & $7.8 \pm 0.4$ & $11.8 \pm 0.7$ \\
\hline 0.200 & 8 & $20.1 \pm 0.2$ & $22.4 \pm 0.1$ & $18.4 \pm 1.1$ & $6.3 \pm 0.4$ & $11.8 \pm 0.7$ \\
\hline 0.250 & 10 & $18.1 \pm 0.2$ & $22.0 \pm 0.1$ & $15.5 \pm 0.9$ & $3.8 \pm 0.2$ & $11.5 \pm 0.7$ \\
\hline 0.350 & 14 & $16.8 \pm 0.2$ & $21.5 \pm 0.1$ & $14.4 \pm 0.8$ & $1.8 \pm 0.1$ & $11.4 \pm 0.7$ \\
\hline 0.375 & 15 & & $21.1 \pm 0.1$ & $12.6 \pm 0.7$ & $1.0 \pm 0.1$ & $11.4 \pm 0.7$ \\
\hline 0.500 & 20 & & $21.0 \pm 0.1$ & $11.1 \pm 0.7$ & & $11.1 \pm 0.7$ \\
\hline 0.750 & 30 & & $21.0 \pm 0.1$ & $9.7 \pm 0.6$ & & $9.7 \pm 0.6$ \\
\hline
\end{tabular}

$\mathrm{T}_{\mathrm{m} 1}$ and $\mathrm{T}_{\mathrm{m} 2}$, transition temperatures of the first and second DSC-peak; $\Delta \mathrm{H}_{\text {cal total }}$, total transition enthalphy; $\Delta \mathrm{H}_{\mathrm{cal1}}$ and $\Delta \mathrm{H}_{\mathrm{cal2}}$, transition enthalpies of the first and second DSC-peak. 
Table 2. Phase transition temperature $T_{m}$ of DMPC-vesicles as a function of the surfactin concentration determined by static light scattering measurements

\begin{tabular}{|c|c|c|c|c|c|}
\hline \multicolumn{2}{|c|}{ Surfactin concentration } & \multirow{2}{*}{$\begin{array}{c}\mathrm{T}_{\mathrm{m} 1 \text {-down }} \\
{ }^{\circ} \mathrm{C}\end{array}$} & \multirow{2}{*}{$\begin{array}{c}\mathrm{T}_{\mathrm{m} 2 \text {-down }} \\
{ }^{\circ} \mathrm{C}\end{array}$} & \multirow{2}{*}{$\begin{array}{l}\mathrm{T}_{\mathrm{ml} \text {-up }} \\
{ }^{\circ} \mathrm{C}\end{array}$} & \multirow{2}{*}{$\begin{array}{c}\mathrm{T}_{\mathrm{m} 2 \text {-up }} \\
{ }^{\circ} \mathrm{C}\end{array}$} \\
\hline $\mathrm{mM}$ & $\mathrm{mol} \%$ & & & & \\
\hline 0.000 & 0 & $23.4 \pm 0.1$ & & $23.6 \pm 0.1$ & \\
\hline 0.025 & 1 & $23.3 \pm 0.1$ & & $23.6 \pm 0.1$ & \\
\hline 0.050 & 2 & $23.1 \pm 0.1$ & & $23.5 \pm 0.1$ & \\
\hline 0.075 & 3 & $22.2 \pm 0.1$ & & $23.0 \pm 0.1$ & \\
\hline 0.100 & 4 & $22.0 \pm 0.1$ & $23.5 \pm 0.1$ & $22.5 \pm 0.1$ & $26.2 \pm 0.2$ \\
\hline 0.125 & 5 & $21.2 \pm 0.1$ & $22.6 \pm 0.1$ & $22.0 \pm 0.1$ & $25.5 \pm 0.2$ \\
\hline 0.150 & 6 & $20.9 \pm 0.1$ & $22.9 \pm 0.1$ & $21.4 \pm 0.1$ & $25.5 \pm 0.2$ \\
\hline 0.175 & 7 & $19.8 \pm 0.1$ & $23.0 \pm 0.1$ & $21.0 \pm 0.1$ & $25.5 \pm 0.2$ \\
\hline 0.200 & 8 & $19.2 \pm 0.1$ & $24.0 \pm 0.1$ & $20.3 \pm 0.1$ & $25.5 \pm 0.2$ \\
\hline 0.250 & 10 & $19.2 \pm 0.1$ & $24.0 \pm 0.1$ & $19.4 \pm 0.1$ & $24.4 \pm 0.2$ \\
\hline 0.375 & 15 & - & $24.0 \pm 0.1$ & - & $24.4 \pm 0.2$ \\
\hline 0.500 & 20 & & partially & eversible & \\
\hline 0.750 & 30 & & totally & versible & \\
\hline
\end{tabular}

$\mathrm{T}_{\mathrm{m} 1 \text { down }}$ and $\mathrm{T}_{\mathrm{m} 2 \mathrm{down}}$, transition temperatures obtained from the down scan experiment;

$\mathrm{T}_{\mathrm{m} 1 \mathrm{up}}$ and $\mathrm{T}_{\mathrm{m} 2 \mathrm{up}}$, transition temperatures obtained from the up scan experiment. 
Table 3. Relaxation times $\tau_{\text {down }}$ and $\tau_{\text {up }}$ obtained from the kinetics of the light scattering of

DMPC-vesicles as a function of the surfactin concentration in the „Poor Man's“ temperaturejump experiment for the down- and up-jump

\begin{tabular}{cccc}
\hline \multicolumn{2}{c}{ Surfactin } & $\tau_{\text {down jump }}$ & $\tau_{\text {up jump }}$ \\
$\mathbf{m M}$ & $\mathbf{m o l} \%$ & $\mathrm{~s}$ & $\mathrm{~s}$ \\
\hline 0.000 & 0 & $29.8 \pm 1.5$ & $29.8 \pm 1.5$ \\
0.025 & 1 & $24.2 \pm 1.2$ & $24.8 \pm 1.2$ \\
0.050 & 2 & $24.1 \pm 1.2$ & $24.3 \pm 1.2$ \\
0.075 & 3 & $22.0 \pm 1.1$ & $22.0 \pm 1.1$ \\
0.100 & 4 & not determinable & $18.5 \pm 0.9$ \\
\hline
\end{tabular}

Light scattering: wavelength of the incident beam: $350 \mathrm{~nm}$; scattering angle: $90^{\circ}$. 